\title{
The Magellanic Clouds Photometric Survey
}

\author{
Dennis Zaritsky, Eva K. Grebel, Jason Harris \\ UCO/Lick Observatory, UCSC, Santa Cruz, CA, 95064, USA
}

\section{Ian Thompson}

Carnegie Observatories, 813 Santa Barbara St., Pasadena, CA, 91101, $U S A$

\begin{abstract}
We present a brief description of the Magellanic Clouds Photometric Survey. In particular, we discuss examples of how the new generation of densely populated CMDs provide new information than that available previously.
\end{abstract}

\section{Introduction}

The Magellanic Clouds Photometric Survey is a digital UBVI survey of the central $8^{\circ} \times 8^{\circ}$ of the Large Cloud and $4^{\circ} \times 4^{\circ}$ of the Small Cloud. The data will help address a variety of issues and provide, among other products, a differential reddening map, cluster census (which will include estimates of richness, morphology, and age), and a map of the field star formation history.

\section{Observations and Data Reduction}

The data are acquired using the 40-inch Swope telescope at Las Campanas and the Great Circle Camera (Zaritsky, Shectman, \& Bredthauer 1996). The pixel scale is $0^{\prime \prime} 7 \mathrm{~mm}^{-1}$ and the typical seeing is around $1^{\prime \prime} .5$. The observing mode is drift scanning and we produce scans that are $\sim 2^{\circ} \times 24^{\prime}$. The effective exposure time as any one object drifts across the field of view is about 4 minutes. Each scan overlaps adjacent scans, which provides comparison data with which to verify astrometry and photometry. The survey will require 40 clear nights for completion and roughly half is done.

The scans are reduced using an automated pipeline constructed around DAOPHOT (Stetson 1987). The basic description of the reduction procedure is presented by Zaritsky, Harris, \& Thompson (1997). The stellar catalog is $50 \%$ complete at $\mathrm{V}=21.5$ (although this limit is highly sensitive to crowding). Astrometric uncertainties are generally $\leq 0.5$, sufficiently good for multiobject spectroscopy.

The catalog contains RA, Dec, U, $\sigma_{U}, \mathrm{~B}, \sigma_{B}, \mathrm{~V}, \sigma_{V}$, I, and $\sigma_{I}$. A star is only included in the catalog if both $\mathrm{B}$ and $\mathrm{V}$ magnitudes are measured. $\mathrm{U}$ and $\mathrm{I}$ magnitudes are not required, and fewer stars (generally around 50 to $75 \%$ of the total) have U or I magnitudes. The photometry is placed on the Landolt system 


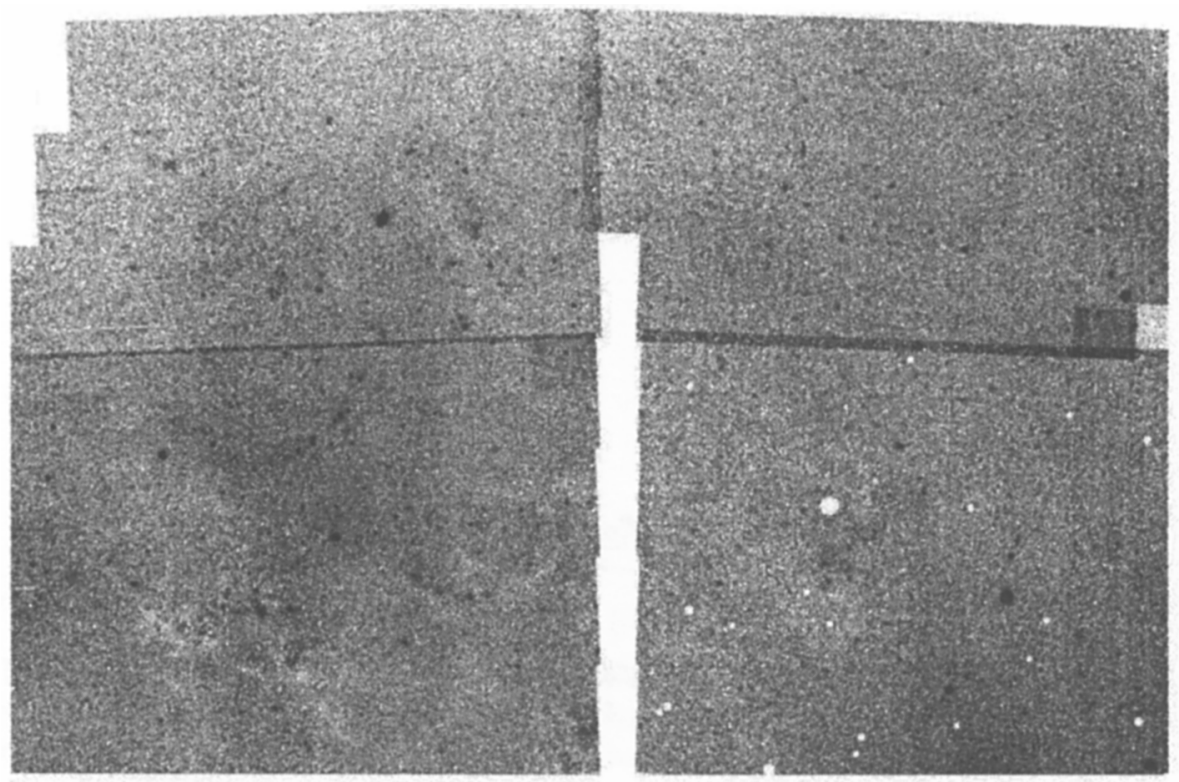

Figure 1. The stellar density within the area of the LMC for which we have reduced data. The $4^{\circ} \times 4^{\circ}$ region lies directly north of the bar ( $\mathrm{N}$ up, $\mathrm{E}$ left). Darker pixels correspond to denser regions. White circles are areas in which saturated stars have been removed.

(Landolt 1992) and astrometry is derived from a comparison to the Magellanic Catalogue of Stars (Tucholke et al. 1996).

\section{Discussion}

So far, we have reduced data from an area of $\sim 4^{\circ} \times 4^{\circ}$ in the LMC that is located above the bar. Figure 1 shows the stellar density map of that region based on 4 million stars with measured B and $\mathrm{V}$ magnitudes (and $V<21$ ). The most visible structure is the Constellation III/LMC IV region (in the middle of the left side of the Figure). Some artifacts of the survey are also evident (there are (1) some horizontal and vertical stripes of higher density illustrating regions where stars from overlapping scans have not yet been matched, (2) some gaps between scans where the data from the first year was judged to be of unusable quality, and (3) a few small patches, such as in the far right of the area, where a problem with the reduction pipeline caused an overdensity or underdensity of identified stars). All of these artifacts are easily removed, but illustrate some of the quality-control issued raised in such a survey.

The Hess diagram for the stars in Figure 1 is presented in Figure 2. The major features (upper main sequence, red clump, and red giant branch) are visible in the left panel, while more subtle features (red supergiants, foreground disk stars and blue supergiants) can be seen in the right panel. Color-magnitude 

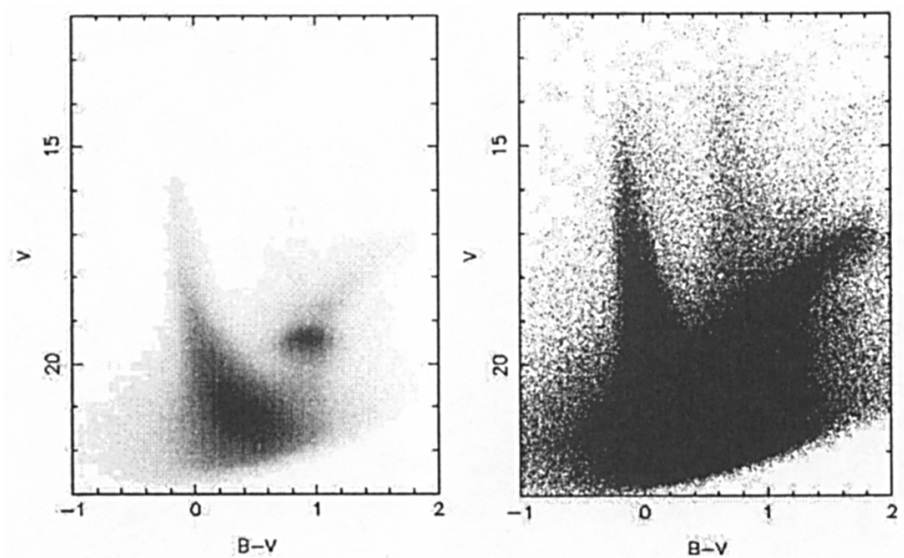

Figure 2. The B-V Hess diagram (low contrast on the left and high contrast on the right) based on 4 million stars in the region shown in Figure 1.

diagrams constructed from such a large number of stars illuminate poorly populated phases of stellar evolution and therefore not only provide information on the nature of stellar populations in the Clouds, but also empirical constraints on our theoretical understanding of stellar evolution. For example, a weak clump is seen on the giant branch above the red clump (termed the RGBB, Zaritsky \& Lin 1997, but identified with a evolutionary phase on the AGB, Gallart 1998).

A second feature noted by Zaritsky \& Lin was the vertical extension of the red clump, termed the VRC. The existence of this feature has been confirmed (Beaulieu \& Sackett 1998), but the interpretation is controversial. Aside from the interesting implications that this feature may have for models of stellar evolution and the star formation history of the LMC, it may also provide information on the line of sight distribution of red clump stars near the LMC, which would in turn affect estimates of the microlensing optical depth to the LMC. Unfortunately, the answer to the nature of the VRC is unlikely to be straightforward.

The two competing interpretations, (1) a foreground distribution of red clump stars (Zaritsky \& Lin 1997) and (2) red clump stars of age $\sim 0.5 \mathrm{Gyr}$ (Beaulieu \& Sackett 1998) make differing predictions. The latter predicts that the VRC stars should have characteristics similar to upper main sequence stars (e.g., low velocity dispersion and strong correlation with sites of recent star formation). Using the data presented in Figure 1, we can test whether the VRC stars are distributed like the $\sim 0.5$ Gyr old main sequence stars by calculating the ratio of the two populations (Figure 3). The behavior of the ratio with local density of main sequence stars suggests that some fraction of the VRC stars traces the main sequence stars (since the ratio does not asymptotically approach zero), but that the remainder do not trace the main sequence stars (since the number of VRC stars is independent of main sequence stellar density as the 


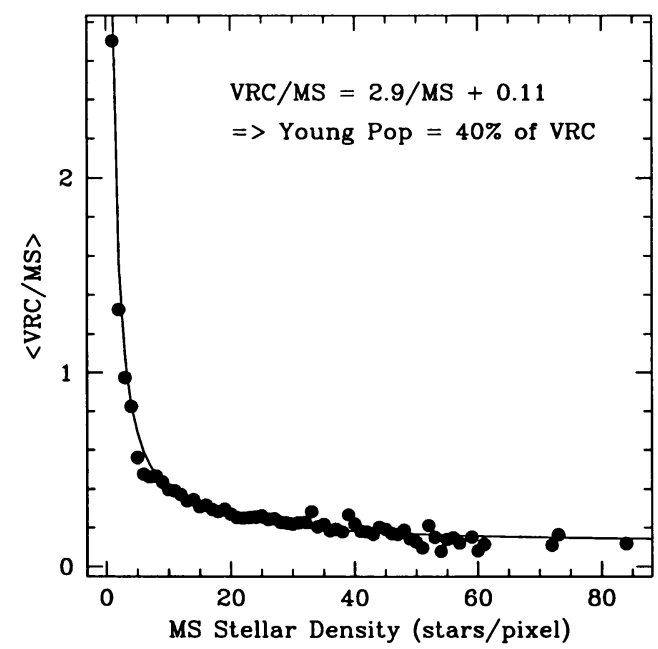

Figure 3. The ratio of VRC stellar density to main sequence stars with $19<V<19.5$. We fit a two-component model where the two components are (1) a uniformly distributed VRC and (2) a directly correlated VRC component.

density approaches zero). A simple model suggests that $60 \%$ of the VRC stars may be unassociated with recent star formation. Supporting evidence for this is given by the large velocity dispersion $\left(\sim 20 \mathrm{~km} \mathrm{~s}^{-1}\right)$ measured for the VRC stars (Zaritsky et al. 1998), which is consistent with a stellar population of several Gyr age (Meatheringham et al. 1988). Therefore, neither hypothesis on the nature of the VRC appears to account for the entire population. Nevertheless, the large databases produced by surveys like the Magellanic Cloud Photometric Survey are invaluable in discovering such features and will be critical in determining their nature.

Acknowledgments. We gratefully acknowledge financial support from a variety of sources (NSF grant AST-9619576, NASA LTSA grant NAG-5-3501, the Dudley Observatory, the Packard Foundation, and the Sloan Foundation).

\section{References}

Beaulieu, J.-P., \& Sackett, P.D. 1998, AJ, 116, 209

Gallart, C. 1998, ApJ, 495, 43

Landolt, A.U. 1992, AJ, 104, 340

Meatheringham, S.J., et al. 1988, ApJ, 327, 651

Stetson, P.B. 1987, PASP, 99, 191

Tucholke, H.-J., de Boer, K.S., \& Seitter, W.C. 1996, A\&AS, 119, 91

Zaritsky, D., Harris, J., \& Thompson, I. 1997, AJ, 114, 1002

Zaritsky, D., \& Lin, D.N.C. 1997, AJ, 114, 2545

Zaritsky, D., Shectman, S.A., \& Bredthauer, G. 1996, PASP, 108, 104 